Network Working Group

Request for Comments: 1525

Obsoletes: 1286

Category: Standards Track
E. Decker cisco systems, Inc. K. McCloghrie Hughes LAN Systems, Inc. P. Langille

$\mathrm{DEC}$

A. Rijsinghani DEC

September 1993

\title{
Definitions of Managed Objects for Source Routing Bridges
}

Status of this Memo

This RFC specifies an Internet standards track protocol for the Internet community, and requests discussion and suggestions for improvements. Please refer to the current edition of the "Internet Official Protocol standards" for the standardization state and status of this protocol. Distribution of this memo is unlimited.

Table of Contents

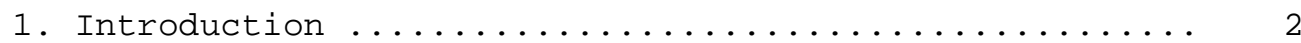

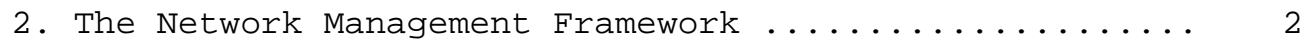

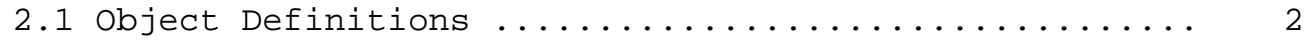

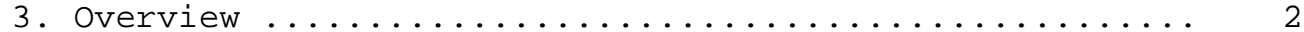

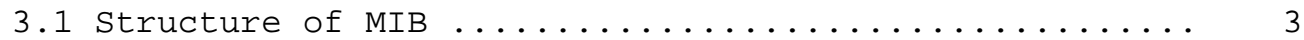

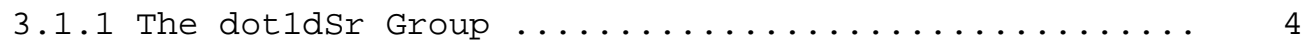

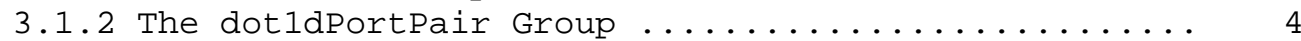

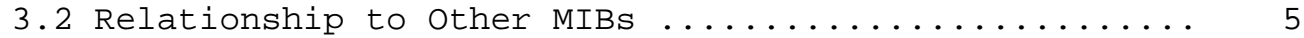

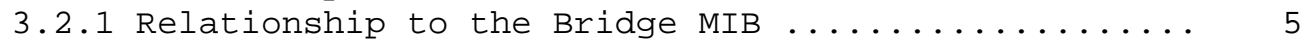

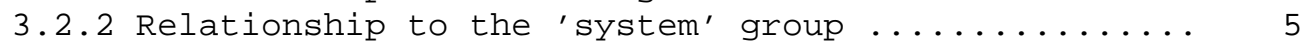

3.2.3 Relationship to the 'interfaces' group .......... 5

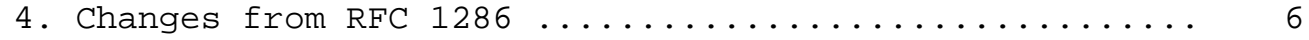

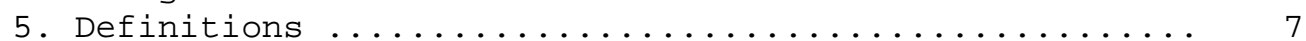

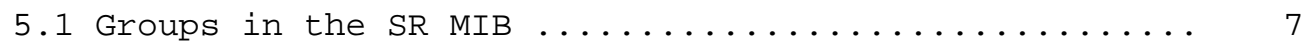

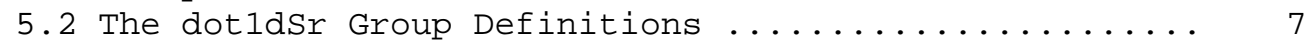

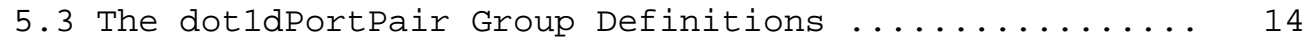

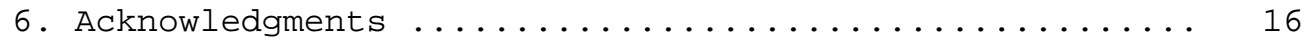

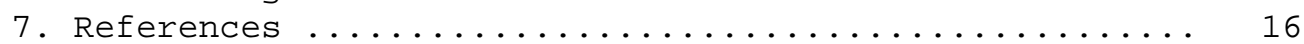

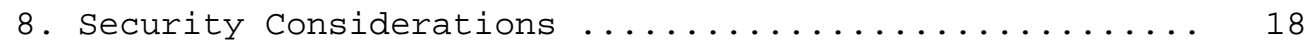

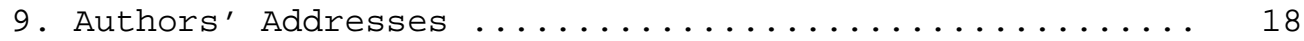




\section{Introduction}

This memo defines a portion of the Management Information Base (MIB) for use with network management protocols in TCP/IP based internets. In particular, it defines objects for managing source routing and source routing transparent bridges. These bridges are also required to implement relevant groups in the Bridge MIB [6].

This MIB supersedes the dot1dSr group of objects published in an earlier version of the Bridge MIB, RFC 1286. Changes have primarily been made to track changes in the IEEE 802.5M SRT Addendum to the IEEE 802.1D Standard for MAC Bridges.

2. The Network Management Framework

The Internet-standard Network Management Framework consists of three components. They are:

- STD 16, RFC 1155 which defines the SMI, the mechanisms used for describing and naming objects for the purpose of management. STD 16, RFC 1212 defines a more concise description mechanism, which is wholly consistent with the SMI.

- STD 17, RFC 1213 defines MIB-II, the core set of managed objects for the Internet suite of protocols.

- STD 15, RFC 1157 which defines the SNMP, the protocol used for network access to managed objects.

The Framework permits new objects to be defined for the purpose of experimentation and evaluation.

\subsection{Object Definitions}

Managed objects are accessed via a virtual information store, termed the Management Information Base or MIB. Objects in the MIB are defined using the subset of Abstract Syntax Notation One (ASN.1) defined in the SMI. In particular, each object object type is named by an OBJECT IDENTIFIER, an administratively assigned name. The object type together with an object instance serves to uniquely identify a specific instantiation of the object. For human convenience, we often use a textual string, termed the descriptor, to refer to the object type.

\section{Overview}

A common device present in many networks is the Bridge. This device is used to connect Local Area Network segments below the network 
layer. There are two major modes defined for this bridging; transparent and source route. The transparent method of bridging is defined in the IEEE 802.1d MAC Bridge specification [11]. Source route bridging has been defined by I.B.M. and is described in the Token Ring Architecture Reference [12], as well as the IEEE $802.5 \mathrm{M}$ SRT Bridge Operations Addendum [14] to 802.1d. This memo defines objects needed for management of a source routing bridge, and is an extension to the SNMP Bridge MIB [6].

An explicit attempt was made to keep this MIB as simple as possible. This was accomplished by applying the following criteria to objects proposed for inclusion:

(1) Start with a small set of essential objects and add only as further objects are needed.

(2) Require objects be essential for either fault or configuration management.

(3) Consider evidence of current use and/or utility.

(4) Limit the total of objects.

(5) Exclude objects which are simply derivable from others in this or other MIBs.

(6) Avoid causing critical sections to be heavily instrumented. The guideline that was followed is one counter per critical section per layer.

\subsection{Structure of MIB}

Objects in this MIB are arranged into groups. Each group is organized as a set of related objects. The overall structure and assignment of objects to their groups is shown below. Where appropriate, the corresponding management object name found in IEEE 802.1d [11] and IEEE 802.5M [14] is also included.

SR Bridge MIB Name

$\operatorname{dot} 1 \mathrm{dSr}$

PortTable

Port

Hopcount

Localsegment

BridgeNum

Targetsegment
IEEE Name

$$
\begin{gathered}
\text { SourceRoutingPort } \\
\text {. Port HopCount } \\
\text {. Segment Number } \\
\text {. BridgeNumber }
\end{gathered}
$$




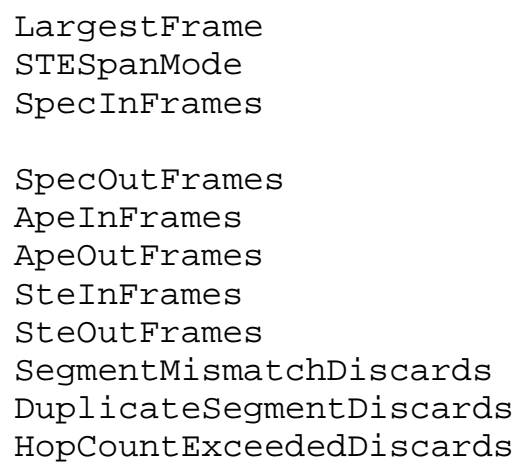

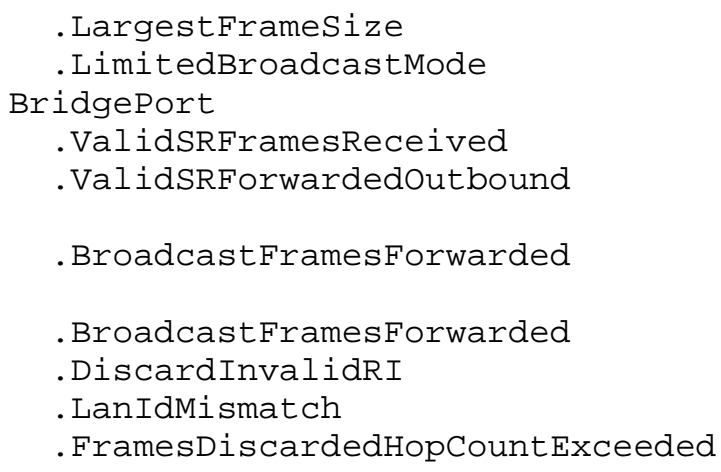

The following IEEE management objects have not been included in the SR Bridge MIB for the indicated reasons.
IEEE Object
Disposition

SourceRoutingPort

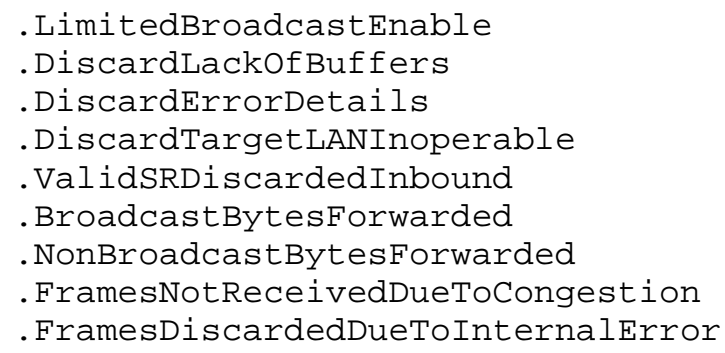

\subsubsection{The dot1dSr Group}

This group contains the objects that describe the entity's state with respect to source route bridging. If source routing is not supported, this group will not be implemented. This group is applicable to source route only, and SRT bridges.

3.1.2. The dot1dPortPair Group

Implementation of this group is optional. This group is implemented by those bridges that support the port-pair multiport model of the source route bridging mode as defined in the IEEE 802.5M SRT Addendum to $802.1 d$. 


\subsection{Relationship to Other MIBs}

As described above, some IEEE 802.1d management objects have not been included in this MIB because they overlap with objects in other MIBs applicable to a bridge implementing this MIB. In particular, it is assumed that a bridge implementing this MIB will also implement (at least) the Bridge MIB and the 'system' group and the 'interfaces' group defined in MIB-II [4].

\subsubsection{Relationship to the Bridge MIB}

The Bridge MIB [6] must be implemented by all bridges, including transparent, SR and SRT bridges. The SR bridge MIB is an extension to the Bridge MIB.

\subsubsection{Relationship to the 'system' group}

In MIB-II, the 'system' group is defined as being mandatory for all systems such that each managed entity contains one instance of each object in the 'system' group. Thus, those objects apply to the entity as a whole irrespective of whether the entity's sole functionality is bridging, or whether bridging is only a subset of the entity's functionality.

\subsubsection{Relationship to the 'interfaces' group}

In MIB-II, the 'interfaces' group is defined as being mandatory for all systems and contains information on an entity's interfaces, where each interface is thought of as being attached to a 'subnetwork'. (Note that this term is not to be confused with 'subnet' which refers to an addressing partitioning scheme used in the Internet suite of protocols.) The term 'segment' is used in this memo to refer to such a subnetwork.

Implicit in this MIB is the notion of ports on a bridge. Each of these ports is associated with one interface of the 'interfaces' group, and in most situations, each port is associated with a different interface. However, there are situations in which multiple ports are associated with the same interface. An example of such a situation would be several ports, each corresponding one-to-one with several X.25 virtual circuits, but all on the same interface.

Each port is uniquely identified by a port number. A port number has no mandatory relationship to an interface number, but in the simple case, a port number will have the same value as the corresponding interface's interface number. 
Some entities provide other services in addition to bridging with respect to the data sent and received by their interfaces. In such situations, only a subset of the data sent/received on an interface is within the domain of the entity's bridging functionality. This subset is considered to be delineated according to a set of protocols, with some protocols being bridged, and other protocols not being bridged. For example, in an entity which exclusively performed bridging, all protocols would be considered as being bridged, whereas in an entity which performed IP routing on IP datagrams and only bridged other protocols, only the non-IP data would be considered as being bridged.

Thus, this MIB (and in particular, its counters) are applicable only to that subset of the data on an entity's interfaces which is sent/received for a protocol being bridged. All such data is sent/received via the ports of the bridge.

4. Changes from RFC 1286

In addition to being separated from the Bridge MIB into a separate document, the following changes were implemented as a result of feedback from IEEE 802.5M:

(1) Changed syntax of dot1dSrPortLargestFrame to INTEGER in order to allow for having 64 possible values as described in draft 7 of the SR Addendum. Listed all legal values in description.

(2) Updated syntax of dot1dSrPort, used to index into dot1dSrPortTable, to use the range (1..65535).

(3) Added a counter to dot1dSrPortTable to count occurrences of duplicate LAN IDs or Tree errors.

(4) Added a counter to dot 1 dSrPortTable to count LAN ID mismatches.

(5) Added text to dot1dSrPortspecInframes and dot1dSrPortSpecoutFrames clarifying that they are also referred to as Source Routed Frames.

(6) Added text to dot1dSrPortApeInFrames and dotldSrPortApeOutFrames clarifying that they are also referred to as All Routes Explorer frames.

(7) Added a scalar variable to the dotldSr group to indicate whether the bridge uses 3 bit or 6 bit length negotiation fields. 
(8) Added dot1dPortPairgroup to allow representation of port pairs as defined in the IEEE 802.5M SRT Addendum.

5. Definitions

SOURCE-ROUTING-MIB DEFINITIONS : := BEGIN

IMPORTS

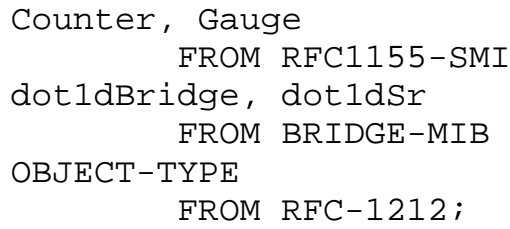

"A list of information for each port of a source route bridge."

INDEX \{ dot1dSrPort \} 


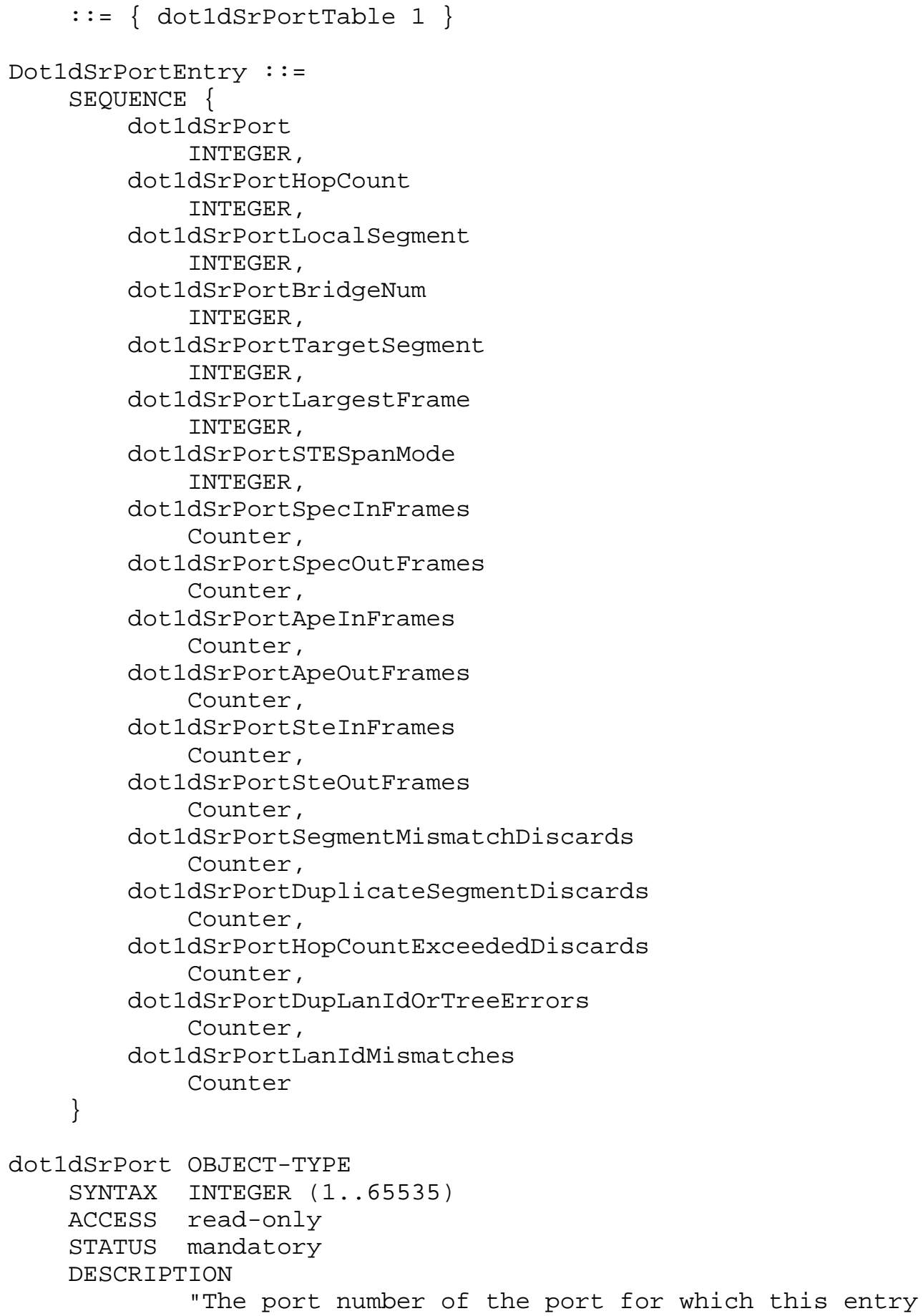




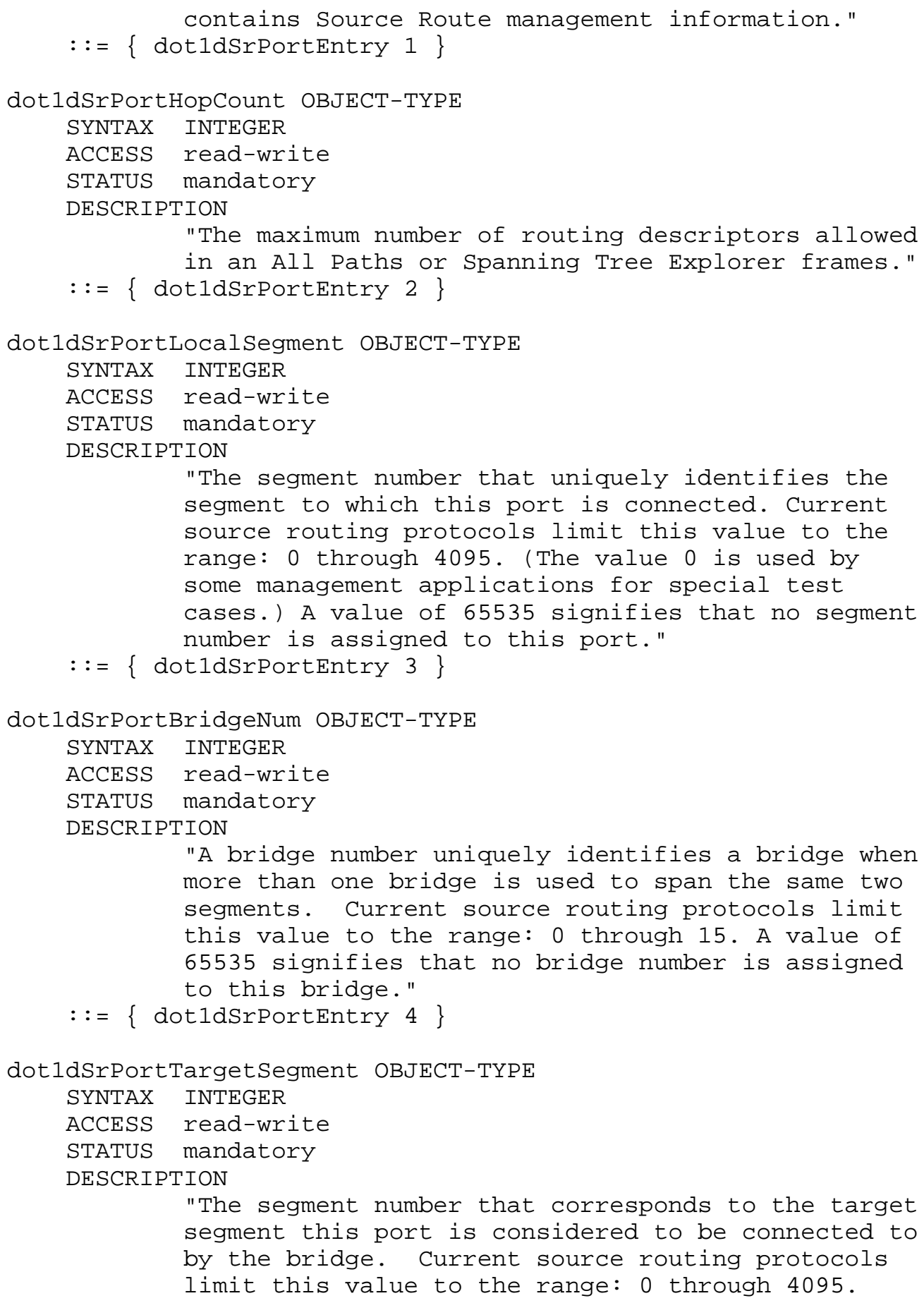


(The value 0 is used by some management applications for special test cases.) A value of 65535 signifies that no target segment is assigned to this port." $::=\{$ dot 1 dSrPortentry 5$\}$

-- It would be nice if we could use ifMtu as the size of the -- largest frame, but we can't because ifMtu is defined to be -- the size that the (inter-) network layer can use which can

-- differ from the MAC layer (especially if several layers of -- encapsulation are used).

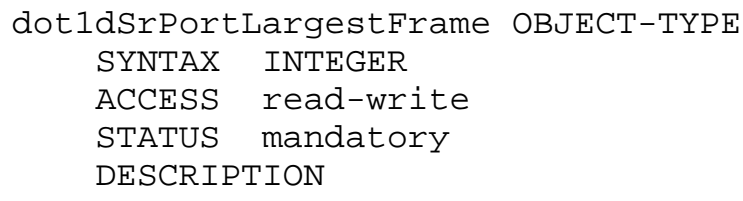

An illegal value will not be accepted by the bridge."

$::=\{$ dot1dSrPortentry 6$\}$

dot1dSrPortSTESpanMode OBJECT-TYPE

SYNTAX INTEGER \{

auto-span (1),

disabled (2),

forced (3)

\}

ACCESS read-write 


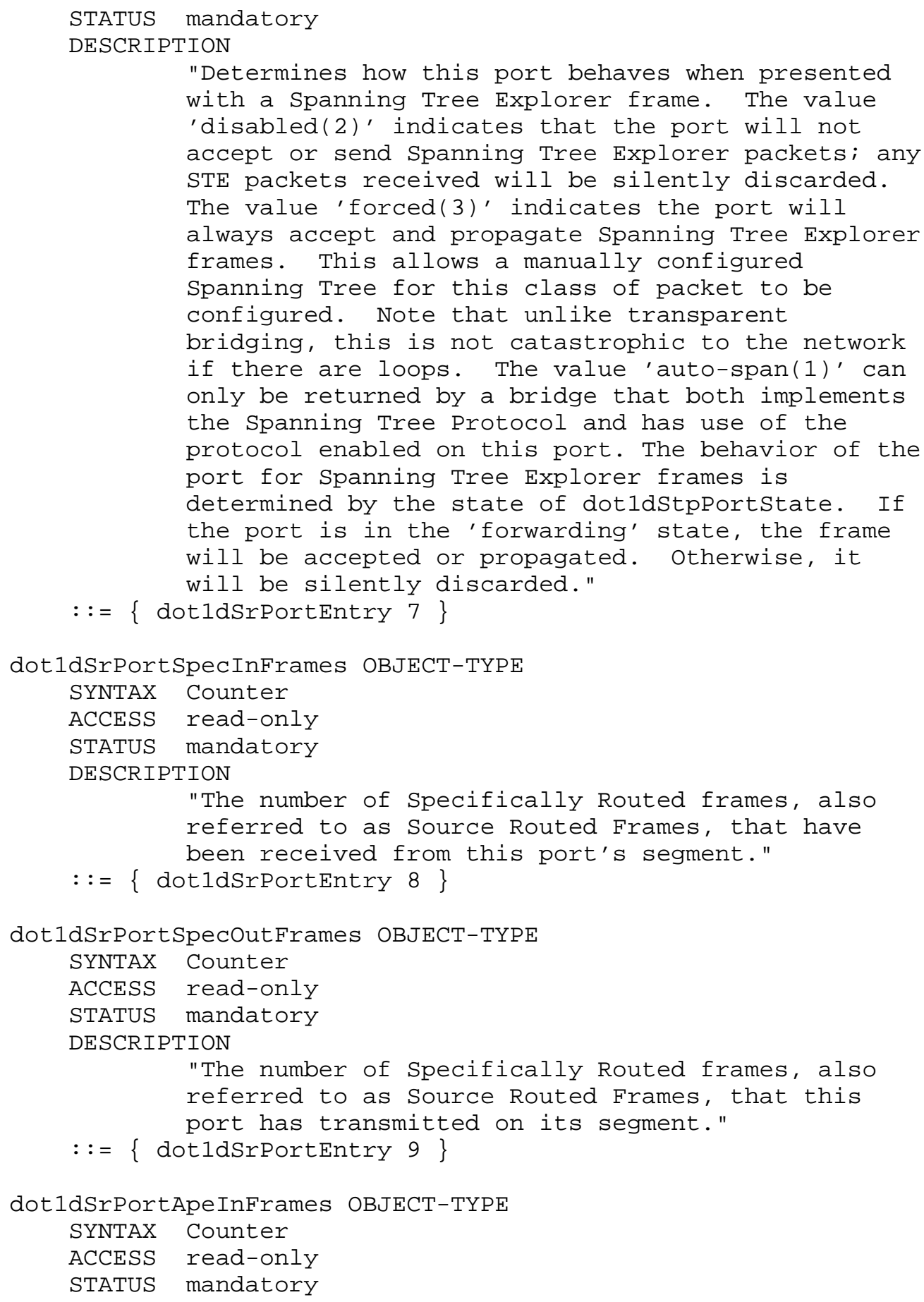




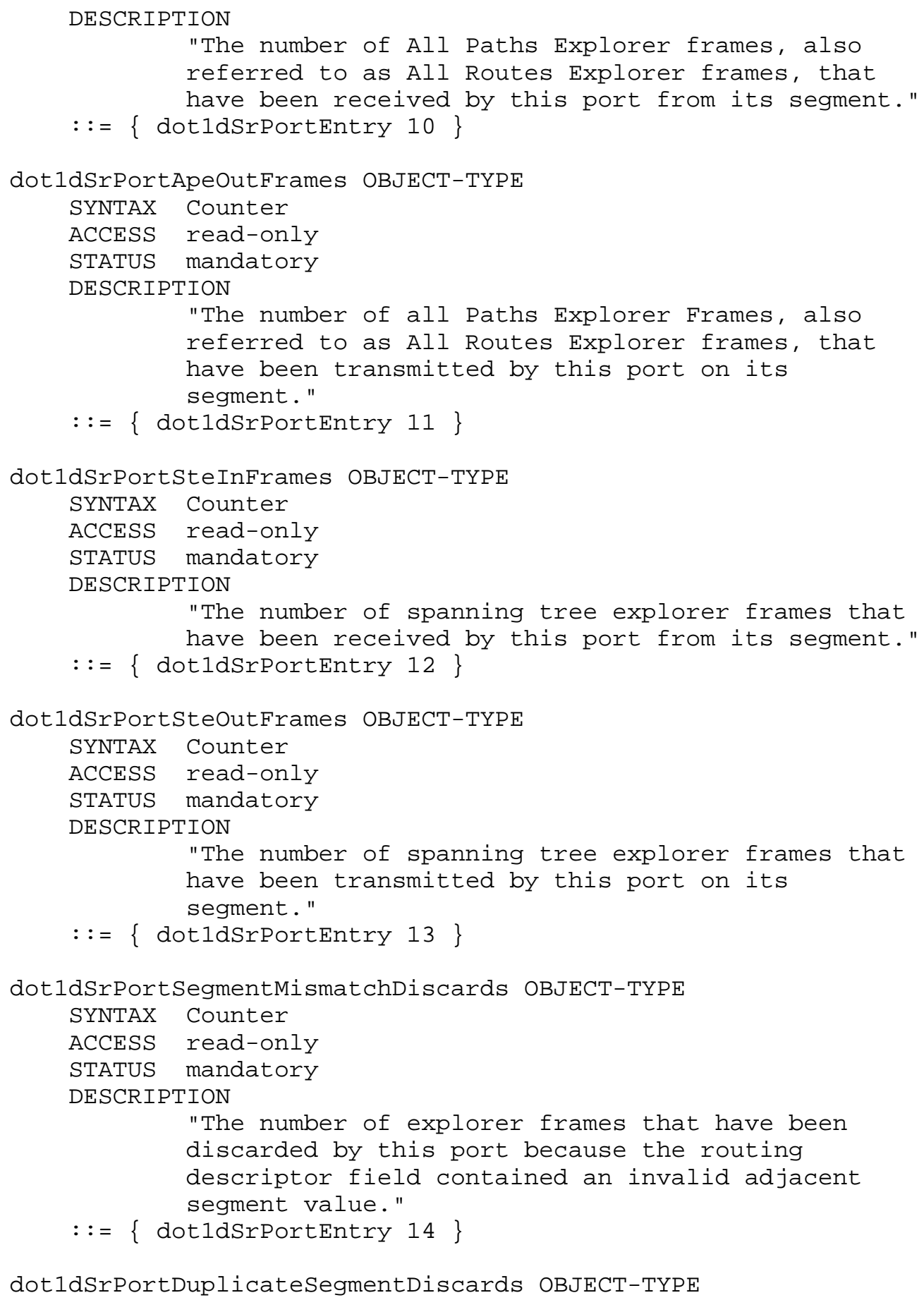




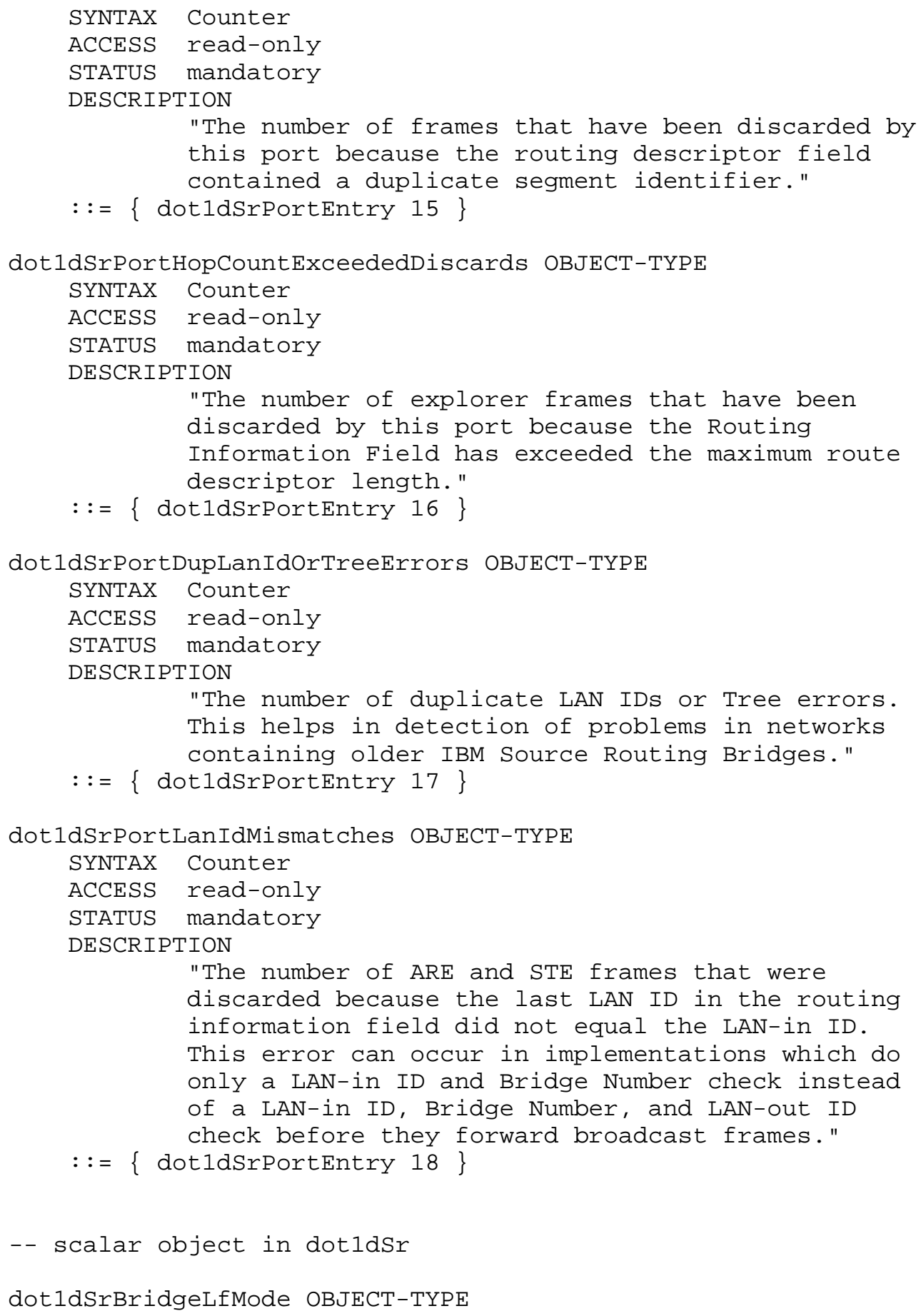




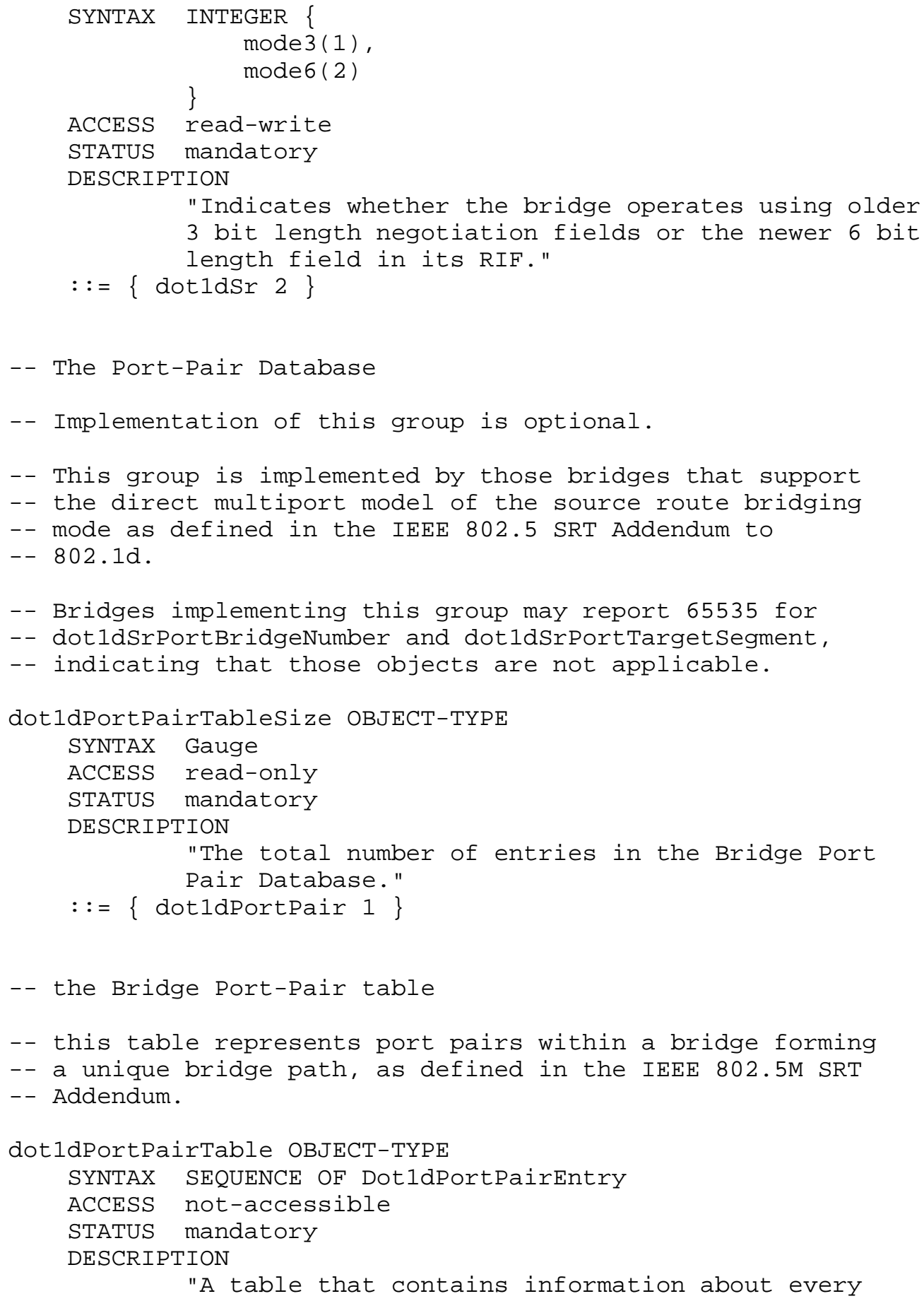

"A table that contains information about every 


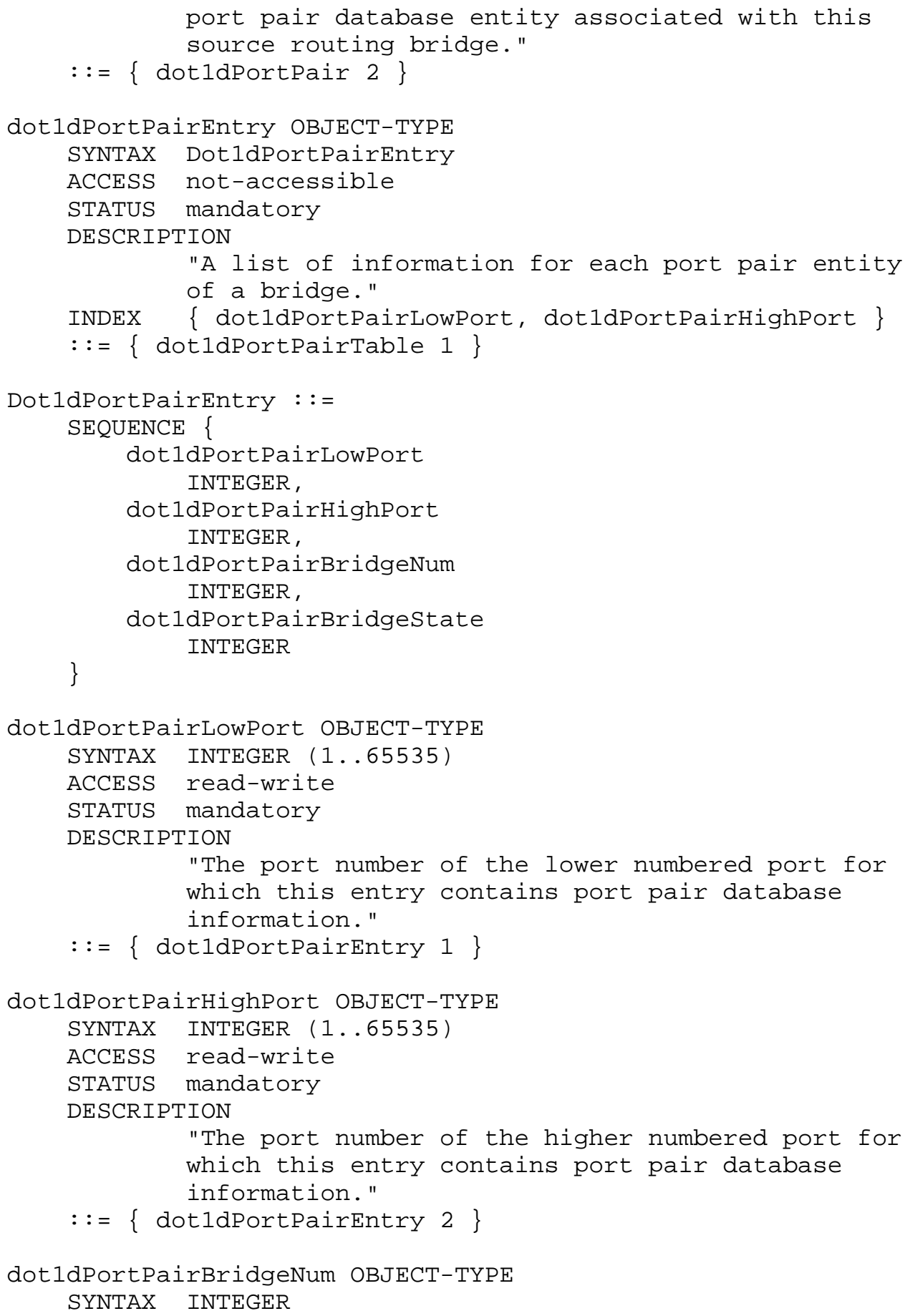

Decker, McCloghrie, Langille \& Rijsinghani 


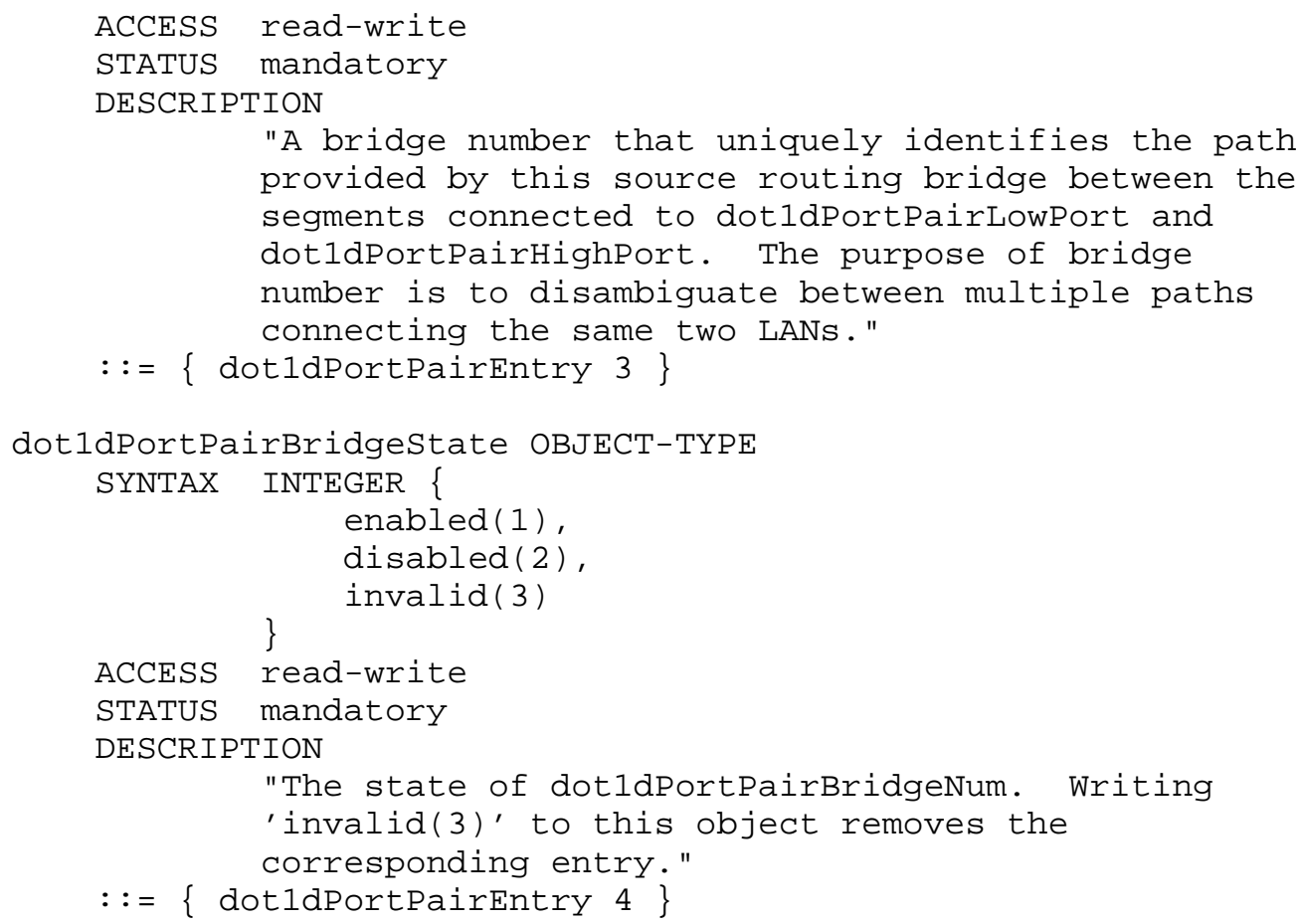

END

6. Acknowledgments

This document was produced on behalf of the Bridge MIB Working Group in the NM area of the Internet Engineering Task Force.

The authors wish to thank the members of the Bridge MIB Working Group for their many comments and suggestions which improved this effort.

7. References

[1] Cerf, V., "IAB Recommendations for the Development of Internet Network Management Standards", RFC 1052, NRI, April 1988.

[2] Cerf, V., "Report of the Second Ad Hoc Network Management Review Group", RFC 1109, NRI, August 1989.

[3] Rose M., and K. McCloghrie, "Structure and Identification of Management Information for TCP/IP-based internets", STD 16, RFC 
1155, Performance Systems International, Hughes LAN Systems, May 1990 .

[4] McCloghrie K., and M. Rose, Editors, "Management Information Base for Network Management of TCP/IP-based internets", STD 17, RFC 1213, Performance Systems International, March 1991.

[5] Case, J., Fedor, M., Schoffstall, M., and J. Davin, "Simple Network Management Protocol", STD 15, RFC 1157, SNMP Research, Performance Systems International, Performance Systems International, MIT Laboratory for Computer Science, May 1990.

[6] Decker, E., Langille, P., Rijsinghani, A., and McCloghrie, K., "Definitions of Managed Objects for Bridges", RFC 1493, cisco Systems, Digital Equipment Corporation, Digital Equipment Corporation, Hughes LAN Systems, July 1993.

[7] Information processing systems - Open systems Interconnection Specification of Abstract Syntax Notation One (ASN.1), International Organization for Standardization, International Standard 8824, December 1987.

[8] Information processing systems - Open systems Interconnection Specification of Basic Encoding Rules for Abstract Notation One (ASN.1), International Organization for Standardization, International Standard 8825, December 1987.

[9] Rose, M., and K. McCloghrie, Editors, "Concise MIB Definitions", STD 16, RFC 1212, Performance Systems International, Hughes LAN Systems, March 1991.

[10] Rose, M., Editor, "A Convention for Defining Traps for use with the SNMP", RFC 1215, Performance Systems International, March 1991.

[11] ANSI/IEEE Standard 802.1D-1990 MAC Bridges, IEEE Project 802 Local and Metropolitan Area Networks, (March 8, 1991).

[12] I.B.M. Token Ring Architecture Reference.

[13] ISO DIS 10038 MAC Bridges.

[14] ANSI/IEEE P802.5M-Draft 7, "Source Routing Transparent Bridge Operation", IEEE Project 802 (1991).

[15] ANSI/IEEE 802.1y, "Source Routing Tutorial for End System Operation", (September, 1990). 


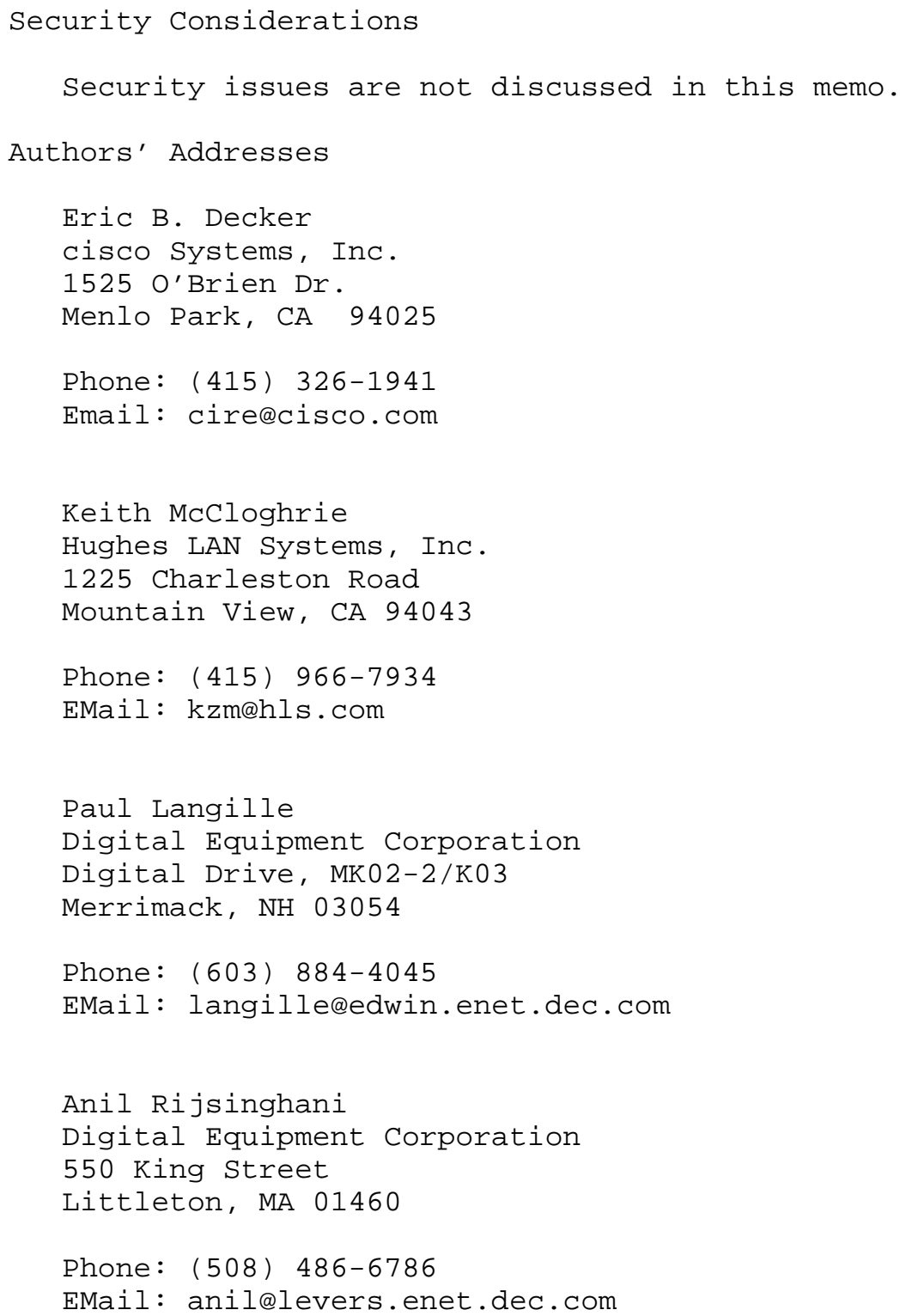

